\title{
Validez del voto en blanco en Chile: un análisis crítico
}

\author{
Viviana Ponce de León Solís*
}

\begin{abstract}
RESUMEN
A lo largo de las últimas décadas ha surgido un creciente interés en torno al significado y valor del voto en blanco. Este último ya no es visto como una mera preferencia electoral no expresada, sino como una señal de protesta y de descontento político. En este contexto, la experiencia comparada da cuenta de una clara tendencia en orden a reconocer institucionalmente ese descontento y permitir su formulación de manera oficial e inequívoca en los procesos electorales. Chile podría sumarse a esta tendencia, en virtud de un recientemente presentado proyecto que modifica la Carta Fundamental para reconocer validez al voto en blanco en elecciones populares (Boletin 1145307). Esta investigación discute críticamente dicho proyecto desde un punto de vista teórico, a la luz de su potencial impacto en el pluralismo político, la participación electoral y en la gestión de los partidos políticos.
\end{abstract}

Voto en blanco - voto expresivo - democracia

\section{Validity of blank ballots in Chile: a critical review}

\begin{abstract}
During the last decades a growing interest has emerged over the meaning and value of the blank ballot. The latter is no longer seen merely as an unexpressed electoral preference but as sign of protest and of political discontent. In this context, comparative experiences show a clear trend towards an institutional recognition of such discontent and allowing its official and unequivocal articulation in electoral processes. Chile might join this trend by virtue of a recently drafted constitutional amendment project to recognise validity to blank ballots in popular elections (Boletin 11453-07). This research critically discusses such project from a theoretical viewpoint, in light of its potential impact on political pluralism, electoral participation and the governance of political parties.
\end{abstract}

Blank ballot - expressive vote - democracy

* Licenciada en Ciencias Jurídicas y Sociales, Universidad Católica del Norte. Doctora en Derecho, Pontificia Universidad Católica de Valparaíso. Profesora de Derecho Constitucional, Universidad Católica del Norte. Correo electrónico: poncedeleonviviana@gmail.com.

Artículo recibido el 7 de enero de 2018 y aceptado para su publicación en este número el 18 de enero de 2019. 


\section{INTRODUCCIÓN}

$\mathrm{H}$ istóricamente el voto en blanco ha sido visto como una categoría jurídica ambigua, poco interesante y marginal. Ambigua, toda vez que no transmite un mensaje o una señal inequívoca ${ }^{1}$. Poco interesante, en cuanto no suele producir efectos directos y su emisión no suele tener ninguna incidencia en el resultado del respectivo proceso electoral ${ }^{2}$. Marginal, por otro lado, en cuanto se le tiende a entender como un comportamiento político irracional y estadísticamente insignificante ${ }^{3}$.

No obstante, en las últimas décadas ha surgido un creciente interés por la figura del voto en blanco ${ }^{4}$. Este ha pasado de ser visto como una forma de abstención o un tipo de voto inválido a ser considerado una forma de protesta electoral. En ese contexto, a nivel comparado se han instaurado diversos mecanismos tendientes a su reconocimiento jurídico o, al menos se discute tal posibilidad ${ }^{5}$. La idea tras estos mecanismos es brindar a los votantes un canal de expresión inequívoca y oficial de su disconformidad con el sistema de partidos y con los candidatos tradicionales. Con ello, en teoría, se promovería la transparencia en la gestión de dichos partidos y su receptividad a las demandas ciudadanas, se ampliaría el potencial expresivo del voto, se incrementaría la participación electoral y se fomentaría el pluralismo político.

Chile no ha quedado marginado de esta discusión. Recientemente, un grupo de parlamentarios presentó un proyecto de reforma constitucional para reconocer validez al voto en blanco en las elecciones populares de Presidente de la República y de Gobernador Regional. En términos más precisos, el proyecto establece que en cuanto los votos en blanco obtengan la mayoría respecto de los demás candidatos, deba repetirse por una sola vez la elección. En esta segunda elección no podrán presentarse los mismos candidatos que se hayan presentado a la primera.

Así las cosas, el propósito de esta investigación es analizar críticamente la propuesta de reconocer validez al voto en blanco contenida en el proyecto. Para ello, la investigación se estructura del siguiente modo. Primero, se examinan las principales aproximaciones en torno a las motivaciones que subyacen a la emisión de un voto en blanco y se revisan superficialmente los principales argumentos en que se funda la propuesta de su reconocimiento jurídico. Luego, se identifican las principales modalidades de reconocimiento del voto en blanco a nivel comparado. En la selección de los sistemas electorales que ilustran estas modalidades se consideró solo a aquellos que escrutan separadamente los votos en blanco de los votos nulos, con el fin de apreciar los potenciales efectos electorales de los primeros en forma aislada. Finalmente, se discute desde un punto de vista teórico

\footnotetext{
${ }^{1}$ Damore; Waters; Bowlers, 2012, p. 895; Power; Garand, 2007, p. 433.

${ }^{2}$ Galatas, 2008, p. 248.

${ }^{3}$ UGGLA, 2008, p. 1144.

${ }^{4}$ Brown, 2011, pp. 364 s.

${ }^{5}$ Con especial referencia a la creación de "partidos en blanco", que intentan simular los efectos de la atribución de efectos electorales directos al voto en blanco, mediante la no comparecencia de sus miembros al hemiciclo, véase Superti, 2014, p. 12.
} 
el efectivo potencial del arreglo institucional propuesto en el proyecto de reforma para alcanzar los fines a que aspira. En este sentido, la idea central que aquí se plantea es que dicho arreglo no sería el más adecuado para promover el pluralismo político, canalizar disenso político o incentivar la autorreforma de los partidos políticos.

El presente estudio se encuadra dentro de las áreas del derecho constitucional y del derecho electoral, y es desarrollado a partir de una perspectiva teórica. Sin perjuicio de ello, se nutre de ciertas contribuciones teóricas y empíricas de la ciencia política, particularmente en las secciones II y IV. En la sección II, dichas contribuciones permiten esbozar las premisas esenciales en que se funda la noción de voto protesta e identificar superficialmente los argumentos en que implícitamente parece fundarse el proyecto. En la sección IV, a su turno, se invocan los estudios empíricos que, desde el enfoque de la ciencia política, avalan o refutan las observaciones teóricas que se formulan en esta investigación.

\section{SignificAdo Y VALOR DEL VOTO EN BLANCO}

El voto en blanco ha sido definido como "un acto por medio del cual un elector manifiesta en un proceso electoral su inhabilidad o negativa a hacer una elección en una oferta política determinada" ${ }^{6}$. Según se desprende de esta definición, la característica distintiva del voto en blanco es que no contiene una expresión de preferencia por ninguno de los candidatos o partidos indicados en la respectiva papeleta de votación. De ahí que se afirme que constituye un "no voto"7, un "voto no expresado", una categoría "gemela" a la de voto nulo9, "a medio camino entre la participación y la abstención"10, que importa una "abstención activa" o una "abstención participante" 11 . En consonancia con esta caracterización, el tratamiento doctrinario del voto en blanco suele estar centrado en las motivaciones que subyacen a su emisión y al valor que, consecuentemente, se le debe atribuir. Los párrafos que siguen se destinan al desarrollo de estas ideas.

\section{Motivaciones subyacentes al voto en blanco}

Entendido el voto en blanco como un no voto, resulta difícil determinar las verdaderas razones que inspiran su emisión. Mas, en literatura, este punto se ha tratado con un notable prejuicio negativo. Por lo general, se ha estimado que el voto en blanco

\footnotetext{
${ }^{6}$ ZUFILKARPASIC, 2001, p. 247.

${ }^{7}$ Ibarzabal; Laruelle, 2017 , p. 1.

8 ZuFilkarpasic, 2001, p. 247.

${ }^{9}$ UGGLA, 2008, p. 1143 . También, en sentido análogo, Brewer, 1990, p. 94, quien considera al voto en blanco como un tipo de voto que adolece de una causal de nulidad por inexistencia.

${ }^{10}$ Ibid.

${ }^{11}$ La expresión "abstención activa” parece haber sido acuñada por SCHEPIS, 1963, p. 346. Ella también es utilizada como sinónimo de la nomenclatura “abstención participante”. Cfr. Hernández, 2008, p. 357.
} 
no importa un acto consciente y deliberado del elector, sino el resultado de su apatía, confusión o ignorancia ${ }^{12}$. Este prejuicio se enraíza en el modelo downsiano del rational choice, en donde se asume que el comportamiento de los votantes está guiado por sus expectativas respecto de los resultados de la elección y por la consideración de que su voto puede influir en esos resultados. Siendo así, es de esperar que en los procesos electorales los ciudadanos actúen racionalmente y voten por aquel candidato o partido que, a su juicio, pueda proveerles más beneficios que el resto ${ }^{13}$. Dicho lo anterior, las alternativas de comportamiento electoral que razonablemente podrían esperarse de los ciudadanos son $\operatorname{dos}^{14}$. Primero, que emitan un voto en favor de su candidato o partido preferido. $\mathrm{O}$, segundo, que se abstengan, si es que los candidatos o partidos en competencia les resultan indiferentes. Concurrir al local de votación y emitir un voto que no exprese ninguna preferencia, por contraste, sería un comportamiento electoral irracional ${ }^{15}$.

De cara a las crecientes tasas de voto en blanco alrededor del mundo en las últimas décadas ${ }^{16}$, el modelo del voto expresivo proporciona una perspectiva diversa. Para dicho modelo, los votantes obtienen un beneficio directo de la preferencia misma que adoptan, con independencia de los resultados generales de la elección ${ }^{17}$. Estos beneficios, de acuerdo con las diversas variantes de dicha corriente, pueden consistir en confirmar la identidad personal de su emisor ${ }^{18}$, permitir la expresión política de los votantes ${ }^{19}$ o canalizar su protesta política ${ }^{20}$. La ventaja de este modelo estribaría en explicar más satisfactoriamente que el modelo del rational choice por qué algunos votantes concurren a votar y deciden anular su voto o dejarlo en blanco, incluso en sistemas en que el voto no es obligatorio y el costo de la abstención es muy bajo ${ }^{21}$.

En este orden de ideas, el voto en blanco importaría un "voto de protesta" 22 , distinto respecto de otras conductas electorales no convencionales, como la abstención o la anulación del voto. Quien emite un voto en blanco no se excluye a sí mismo de un

\footnotetext{
12 Damore; Waters; Bowlers, 2012, pp. 895-907; Driscoll; Nelson, 2014, pp. 1-15.

${ }^{13}$ Downs, 1957 , p. 36.

${ }^{14} \mathrm{Al}$ menos en los sistemas en que el voto es voluntario o la abstención no conlleva costos adicionales. Ibid., pp. 261-265.

${ }^{15}$ Esta fue la idea en que se inspiró la redacción del actual artículo 15 de la Constitución, como se desprende del siguiente pasaje: "[...] el voto en blanco es casi antinatural y constituye una excepción, y en ese caso el voto emitido carece de todo sentido de responsabilidad”. Cfr. Historia de la Ley. Constitución Política de la República. Artículo 15, Derecho a Sufragio, p. 39.

${ }^{16}$ Cfr. Superti, 2015, p. 6.

17 NúÑEZ, 2016, p. 899.

18 Hillman, 2010, pp. 403-418.

${ }^{19}$ McMurray, 2017, p. 208.

${ }^{20}$ Véanse, con referencias adicionales, Álvarez; Kiewiet; NúÑez, 2018, p. 2; Superti, 2015, p. 4; Zufilkarpasic, 2001, pp. 253 y 264-266.

${ }^{21}$ Esto no implica proponer una sustitución del modelo del rational choice por el modelo de voto expresivo. Pese a que ambos pueden ser vistos como rivales, también pueden ser vistos como explicaciones complementarias de un fenómeno más complejo. Cfr. Brennan; Hamlin, 1998, p. 173.

22 Damore; Waters; Bowlers, 2012, pp. 895-907; Driscoll; Nelson, 2014, pp. 1-15; Engelen, 2007, p. 28; McMurray, 2017, p. 208; SlovaK; VAssil, 2015, p. 464.
} 
proceso electoral en particular ni del sistema político en general, sino que se erige en partícipe de uno y otro ${ }^{23}$. Desde ese punto puede distinguirse claramente de la abstención. Por otro lado, quien emite un voto en blanco no expresa preferencia electoral por ninguno de los candidatos o partidos en competencia. En cambio, quien emite un voto nulo expresa una preferencia electoral, pero lo hace incorrecta o ilegítimamente en cuanto no identifica dicha preferencia de manera singular o anónima ${ }^{24}$. Así, se afirma que el voto en blanco "es un voto que se emite desde la no preferencia (y hasta desde el rechazo) por todas las opciones electorales concurrentes, por todas las candidaturas" 25 .

De aceptarse estas ideas podría incluso decirse que el voto en blanco tiene un valor expresivo más importante que el abstencionismo, pues se adhiere al proceso democrático. El elector se presenta al recinto de votación, soportando todos los costos que ello pueda conllevar, con el fin de enviar un mensaje. Considerando que la alternativa de no ejercer su derecho resulta menos costosa (en términos de tiempo y de dinero) y produce jurídicamente un mismo efecto, es razonable suponer que su decisión es intencional e intencionada. Entonces, ya no se puede considerar que su opción representa un simple "no voto"; antes bien representa un "voto no" 26.

\section{Argumentos en favor del reconocimiento del voto en blanco}

Pese a la ambigüedad que buena parte de los estudios teóricos y empíricos atribuyen al voto en blanco, la concepción de este como un voto de protesta o desaprobación ha impulsado una tendencia en orden a institucionalizarle o reconocerle valor jurídico ${ }^{27}$. Con ello, se asume, los votantes podrían articular de manera clara y oficial su disconformidad política, eliminando la ambigüedad antes aludida ${ }^{28}$. El punto no deja de ser interesante, toda vez que implica una relectura de la democracia representativa. Precisamente, este sistema tradicionalmente reposa en la aceptación por parte de los ciudadanos de la papeleta de votación -incluyendo a los candidatos y partidos que figuran en ella-como su principal herramienta de participación. Mas, ante una insatisfacción generalizada con los candidatos tradicionales y el sistema de partidos, la institucionalización del voto en blanco ofrecería un canal para expresar esa insatisfacción utilizando los mecanismos de participación propios de la democracia representativa.

${ }^{23}$ Cfr. CEA, 2013, p. 76; URdánOZ, 2004, p. 305.

${ }^{24}$ Debido a que la singularidad y el anonimato del voto son las dos condiciones que, a nivel comparado, suelen determinar la nulidad del voto. Cfr. Aldashev; Mastrobuoni, 2016, pp. 1-22.

${ }^{25}$ Hernández, 2008, p. 357 . En idéntico orden de ideas véase Superti, 2015, pp. 9-12.

${ }^{26}$ En este sentido puede leerse el siguiente fragmento de Bobbio: "mientras la abstención del no votante se puede interpretar como un estado de indiferencia ante cualquiera de las alternativas planteadas, la abstención de quien vota en blanco debe interpretarse, en cambio, como un estado de hostilidad ante esas opciones". Cfr. BobBio, 2003, pp. 486 s.

${ }^{27}$ Ibarzabal; Laruelle, 2017, p. 8; Superti, 2015, pp. $6 \mathrm{~s}$.

${ }^{28}$ IbarZabal; Laruelle, 2017, p. 2; Superti, 2015, p. 48. 
Luego, en segundo lugar, algunos autores afirman que el reconocimiento del voto en blanco fomentaría la participación electoral y disminuiría las tasas de abstención ${ }^{29}$. Tal afirmación se basa en considerar que la abstención electoral puede estar motivada por la insatisfacción, o incluso hastío, del electorado con las opciones disponibles. Si esa hipótesis resultara ser correcta, una potencial solución sería ampliar el rango de opciones e incorporar entre ellas una opción puramente expresiva que permita articular el rechazo de los votantes hacia los candidatos o partidos en competencia ${ }^{30}$. Con ello, se atraería a quienes se abstenían previamente de votar, para que concurran a las urnas a emitir un voto en blanco.

Por último, el reconocimiento del voto en blanco es vinculado con un fortalecimiento de la gestión de los partidos políticos y, por consiguiente, del sistema democrático como un todo. Asumiendo que el voto en blanco expresa descontento con las opciones que ofrece la papeleta de votación, su emisión puede ser vista no solo como una suerte de sanción a los partidos políticos ${ }^{31}$, sino también como una importante fuente de información política para estos ${ }^{32}$. Así, con base en esa información, los mismos podrían mejorar sus campañas futuras, optimizar su capacidad de respuesta frente a las demandas del electorado (responsiveness), transparentar su rendición de cuentas (accountability) e impulsar su autorreforma ${ }^{33}$. Todo lo anterior resultaría particularmente cierto en aquellos sistemas en que la opción en blanco, de alcanzar un determinado número de votos, puede significar que un escaño quede vacío, que se invalide el resultado de una elección u que sea necesaria su repetición.

\section{GRAdOS DE RECONOCIMIENTO DEL VOTO EN BLANCO}

A nivel comparado, no es inusual que la valoración jurídica del voto en blanco resulte consistente con su caracterización como una categoría "gemela" del voto nulo o como un voto "a medio camino entre la participación y la abstención”. Así, en algunos países (El Salvador ${ }^{34}$, México $^{35}$, Nicaragua ${ }^{36}$, Uruguay $^{37}$, Venezuela ${ }^{38}$ ) el voto que no

29 Mora, 2012, p. 76; Superti, 2015, p. 14.

30 Chatterjee; Szabó; Ujhelyi, 2017, p. 2.

31 IbARZABAL; LARUELle, 2017, p. 8.

32 SlovaK; VASSIL, 2015, p. 477.

33 Ibid.; Superti, 2015, pp. 47 s.

34 Artículo 253-C inciso $1^{\circ}$ del Código Electoral de El Salvador.

35 Artículo 288 numeral 2 letra a) de la Ley General de Instituciones y Procedimientos Electorales de México.

36 Artículo 125 de la Ley $\mathrm{N}^{\circ} 331$, Ley Electoral de Nicaragua.

37 Véase la Sección IV “De los Escrutinios” de la Ley $N^{\circ} 7.812$, Ley de Elecciones de Uruguay, que solo menciona tres clases de votos: los votos objetados, los votos válidos y los votos nulos. No se contempla ninguna referencia a los votos en que no se ha manifestado una preferencia.

38 Artículos 136 , numerales $1^{\circ}$ y $2^{\circ}$, y 137 , numeral $1^{\circ}$, de la Ley Orgánica de Procesos Electorales de Venezuela. 
contiene expresión de preferencia se estima como un tipo de voto nulo o como una abstención. En otros, como Chile, el voto en blanco es considerado como un tipo de voto no válidamente emitido, pero distinto al voto nulo y computado separadamente de este. Si bien esta consideración no implica atribuir efectos jurídicos al voto en blanco ${ }^{39}$, sí supone un grado de reconocimiento jurídico al mismo. Con todo, en la literatura acerca del tópico, ella no suele ser entendida como una forma de reconocimiento relevante. Al tratar de un mismo modo al voto en blanco y al voto nulo -privándoles de efectos jurídicos-, por más que se les escrute separadamente, resulta muy difícil asignarles un significado diferenciado ${ }^{40}$.

Fuera de estos casos, existen diversos mecanismos de reconocimiento jurídico del voto en blanco que parecen superar la concepción tradicional y ser concordantes con la idea de voto protesta o de voto de desaprobación. Concretamente, el presente apartado se destina al estudio de tres de dichos mecanismos: (1) incorporar el voto en blanco como opción explícita en la papeleta de votación; (2) atribuirle efectos electorales indirectos; y (3) atribuirle efectos electorales directos.

\section{Voto en blanco como opción explícita en la papeleta de votación}

Teóricamente, como ya se ha señalado más arriba, uno de los rasgos distintivos del voto en blanco es que no da cuenta de una preferencia electoral. En la práctica, por el contrario, el voto en blanco sí puede reflejar una preferencia claramente manifestada. Así se puede observar en aquellos Estados que han optado por incorporar en sus papeletas de votación una opción que permita al elector expresar de manera inequívoca su desaprobación hacia todos los candidatos indicados en la papeleta. Dicha opción se puede enunciar con diversas fórmulas. Algunos ejemplos de estas fórmulas incluyen: "ninguno de estos candidatos" (none of these candidates, estado de Nevada en Estados Unidos) ${ }^{41}$, "ninguno de los anteriores" (none of the above, India) ${ }^{42}$ o "voto en blanco" (Colombia). Otra variante consiste en la posibilidad de presentarse en el recinto de votación y expresamente declinar votar (Canadá) ${ }^{43}$. Si bien, por consiguiente, estas declinaciones

${ }^{39}$ Aunque el conteo separado del voto en blanco carezca de consecuencias jurídicas, es posible atribuirle un valor simbólico-político. De hecho, en aquellos países donde opera el sistema de voto obligatorio, el voto en blanco es considerado como uno de los pocos mecanismos oficiales que permitirían a los votantes expresar su desconfianza o descontento, junto con la emisión de un voto nulo o el voto en favor de partidos minoritario. Cfr. Hooghe; Marien; Pauwels, 2009, pp. 245-273. Por otro lado, no puede dejar de señalarse que descontar los votos en blanco del total de votos que se considerarán para efectos de adjudicar representación indudablemente puede tener un efecto en los resultados de la elección. Cfr. BobBio, 2008, p. 486.

40 Power; Garand, 2007, p. 433. Sin perjuicio de ello, una propuesta de modelo analítico para identificar las variables que determinan la emisión de un voto en blanco y no de un voto nulo, y viceversa, puede verse en Troumpounis, 2011, pp. 89-94.

${ }^{41}$ Nev. Rev. Stat. \$293.269 (2015).

${ }^{42}$ La fórmula fue incorporada en 1993 por una decisión de la Corte Suprema de la India. Cfr. People’s Union for Civil Liberties v. Union of India (2013) (10) S.C.C., 1, 42-50.

43 Esta figura (que solo se encuentra disponible en algunas provincias de Canadá, como Alberta, Manitoba y Ontario) es independiente de la posibilidad de recibir la papeleta y emitir un voto sin marca. Cfr. Galatas, 2008, pp. 454 s. 
no importan la emisión de un voto, ellas son contabilizadas autónomamente, en forma separada a los votos nulos y a las abstenciones.

\section{Voto en blanco con efectos electorales indirectos}

El siguiente grado de reconocimiento jurídico del voto en blanco consiste en atribuirle consecuencias electorales indirectas, reconociéndole así una validez limitada. Digo limitada, porque en este caso el voto en blanco no suele incidir decisivamente en la determinación de los candidatos que resultarán electos. En lugar de ello, puede tener una influencia en la participación de los partidos políticos en los procesos electorales, cuando menos en tres ámbitos. Primero, en el acceso de los partidos políticos a cargos de elección popular, por lo general -aunque no exclusivamente- legislativos. Segundo, en la asignación de espacios gratuitos de tiempo de propaganda electoral en medios de comunicación de titularidad pública. Y, tercero, en la distribución de recursos públicos para el financiamiento de campañas electorales.

Un ejemplo del primer supuesto puede verse en algunos sistemas electorales que prevén umbrales electorales o cláusulas de barrera, como el argentino. Allí, según el artículo 35 letra c) I del Código Electoral Nacional ${ }^{44}$, los votos válidos pueden ser de dos tipos: afirmativos o en blanco. Son votos afirmativos "los emitidos en las boletas de sufragio que tienen una marca en el espacio correspondiente a una agrupación política". Un voto se contabiliza como en blanco, en cambio, "cuando la boleta no tenga ninguna marca en el espacio correspondiente para las distintas agrupaciones políticas”. Si bien estos no se consideran para proclamar los ganadores de una elección (donde solo se toman en cuenta los "votos afirmativos válidamente emitidos" ${ }^{45}$ ), sí pueden ser relevantes para determinar quiénes pueden participar en las elecciones. Al efecto, se establece un umbral de $1,5 \%$ de los "votos válidamente emitidos" 46 , entre los que se computan tanto los votos afirmativos como los votos en blanco.

Por lo que respecta al segundo supuesto, una ilustración puede encontrarse en el sistema español. En él, la Ley Orgánica 5/1985, de 19 de junio, del Régimen Electoral General $^{47}$, establece un baremo para la distribución del tiempo gratuito de propaganda electoral en medios de comunicación (emisoras de radio y televisión) de titularidad pública ${ }^{48}$. Dicha distribución es hecha de forma proporcionalmente directa al porcentaje de votos válidamente emitidos que cada partido, federación o coalición haya obtenido, entre los que también se contabilizan los votos en blanco. En relación con este punto conviene destacar que la legislación española reputa como tales exclusivamente a los votos que no contienen expresión de preferencia de ninguna clase, mas no a los que contengan algún tipo de marca o alteración ${ }^{49}$.

\footnotetext{
${ }^{44}$ Ley $\mathrm{N}^{\circ} 19.945$, B.O. 19/12/1972.

45 Artículo 149, Código Electoral Nacional.

46 Artículo 60 bis inciso $2^{\circ}$, ibíd.

47 BOE 20/06/1985.

48 Artículo 64, Ley Orgánica 5/1985, de 19 de junio, del Régimen Electoral General.

49 Artículo 96, ibíd.
} 
Finalmente, el sistema colombiano es representativo del tercer supuesto. En Colombia, al igual que en los dos casos anteriores, el voto en blanco también es válido ${ }^{50}$. El punto es relevante porque el Estado debe contribuir al financiamiento de las campañas electorales de partidos y movimientos políticos, así como de movimientos sociales y grupos significativos de ciudadanos que postulen candidatos a cargos de elección popular. Tal financiamiento asume la forma de un derecho a reposición de gastos de campaña que, en principio, es definido solamente en razón de los votos válidos depositados en favor del respectivo candidato ${ }^{51}$. Sin embargo, el derecho a reposición queda excluido en las campañas para Presidente, Alcaldías y Gobernaciones cuando el candidato hubiere obtenido menos del $5 \%$ de los votos válidos en la elección ${ }^{52}$. Debido a que para que opere la exclusión del derecho a reposición el porcentaje no se calcula sobre los votos válidos depositados en favor del candidato, sino sobre los votos válidos a secas, debe entenderse que se incluyen también los votos en blanco.

\section{Voto en blanco con efectos electorales directos}

El último y más alto grado de reconocimiento jurídico del voto en blanco consiste en atribuirle efectos jurídicos directos en el resultado de un proceso electoral. Usualmente estos efectos consisten en impedir que se declare como vencedor al candidato con más preferencias, en la medida que los votos en blanco emitidos superen en alguna proporción a dichas preferencias y obligar a repetir las elecciones. Además, en los ordenamientos que recogen esta modalidad de reconocimiento puede impedirse a los candidatos que participaron en el primer proceso la posibilidad de volver a postularse. De esta forma, los partidos o coaliciones que hayan participado en el primer proceso y que se encuentren habilitados para participar nuevamente en el segundo, deberán presentar candidatos distintos a los anteriores.

A nivel académico, uno de los más célebres ejemplos de la situación aquí descrita puede encontrarse en Colombia. De acuerdo con el parágrafo $1^{\circ}$ del artículo 258 de la Constitución Política de ese país, “[d]eberá repetirse por una sola vez la votación para elegir miembros de una Corporación Pública, Gobernador, Alcalde o la primera vuelta en las elecciones presidenciales, cuando del total de votos válidos, los votos en blanco constituyan la mayoría" 53 . La misma disposición añade que "[t]ratándose de elecciones unipersonales no podrán presentarse los mismos candidatos, mientras en las de

${ }^{50}$ Así se desprende del artículo 137 del Código Electoral Colombiano (Decreto 2241 de 1986, D.O. No 37571, 01/08/1986), cuyo inciso segundo dispone: “[e]l voto en blanco se tendrá en cuenta para obtener el cuociente electoral".

51 Artículo 13 de la Ley 130 de 1994 (Estatuto Básico de los partidos y movimientos políticos, D.O. $\left.\mathrm{N}^{\circ} 41280,23 / 03 / 1994\right)$.

52 Véanse la letra a) y el inciso 3, respectivamente, del artículo 13 de la Ley 130 de 1994.

53 Aunque la norma no es explícita al respecto, se entiende que se refiere a la mayoría absoluta $(50 \%$ más uno) y no a la mayoría relativa, de acuerdo con la interpretación que la Corte Constitucional de Colombia ha hecho de ella. Cfr. Corte Constitucional, Sentencia C-490-2011. 
Corporaciones Públicas no se podrán presentar a las nuevas elecciones las listas que no hayan alcanzado el umbral". Ese umbral es de $3 \%$ de los votos válidos para el Senado de la República y de $50 \%$ del cuociente electoral (que resulta de dividir el número total de votos válidamente emitidos por el número de cargos a proveer) para las demás corporaciones públicas 54 .

Es del caso señalar que en algunos sistemas el voto en blanco puede producir efectos electorales, aun cuando no se le reconozca validez. Así ocurre, por ejemplo, en Perú. Conforme al artículo 184 de la Constitución Política del Perú de 199355, "[e]l Jurado Nacional de Elecciones declara la nulidad de un proceso electoral, de un referéndum o de otro tipo de consulta popular cuando los votos nulos o en blanco, sumados o separadamente, superan los dos tercios del número de votos emitidos". En tal evento, según el artículo 368 de la Ley Orgánica de Elecciones peruana (Ley No 26859) ${ }^{56}$, "las nuevas elecciones se efectúan en un plazo no mayor de 90 (noventa) días".

La principal diferencia entre el caso de Perú y el de Colombia es que en Perú no existen restricciones respecto de los partidos o candidatos que pueden participar en las nuevas elecciones. Esta diferencia resulta reveladora respecto de la diversa significación que se atribuye al voto en blanco en uno y otro caso. De este modo, en Perú, la inquietud que parece subyacer a los efectos del voto en blanco es asegurar que los resultados de una elección sean efectivamente representativos de la voluntad de la mayoría. Por el contrario, la inquietud subyacente en Colombia parece ser más bien canalizar la disidencia de los electores y traducirla en consecuencias electorales concretas que reflejen fielmente dicha disidencia.

\section{Reconocimiento del voto en Blanco en Chile}

Recapitulando lo expuesto en los apartados anteriores, es usual que en la literatura respecto de esta materia se asuma que las diversas formas de reconocimiento jurídico del voto en blanco conllevarían una promoción de la libertad de expresión de los electores. Ello, a su vez, fomentaría su participación electoral, conduciría a la autorreforma de los partidos políticos y, en definitiva, contribuiría a resolver la crisis de la democracia representativa. Con todo, los efectos favorables que en teoría se atribuyen al reconocimiento del voto en blanco (en cualquiera de sus modalidades) permanecen todavía en el plano de la especulación, ya que no hay muchas experiencias de largo plazo que permitan evaluar con precisión su impacto ${ }^{57}$. A causa de lo anterior, la discusión en torno a este punto amerita una reflexión crítica respecto del verdadero alcance de las consecuencias de una reforma en este sentido. A ello se dedican los párrafos que siguen.

\footnotetext{
${ }^{54}$ Artículo 263 inciso $2^{\circ}$, Constitución Política de Colombia.

55 D.O.E.P. 30/12/1993.

56 D.O.E.P. 01/10/1997.

57 Brown, 2011, p. 365; Hooghe, 2011, p. 271; Driscoll; Nelson, 2014, p. 12.
} 


\section{El valor del voto en blanco en el derecho nacional vigente}

En Chile, el voto en blanco goza de reconocimiento a nivel constitucional y legal. En efecto, la Constitución contempla dos referencias textuales al voto en blanco. La primera se encuentra en el artículo 26 inciso $3^{\circ}$, según este, para efectos de las elecciones de Presidente de la República "los votos en blanco y nulos se considerarán como no emitidos”. La segunda referencia, a su turno, se halla en el artículo 111 inciso $6^{\circ}$, que replica la misma idea respecto de las elecciones de gobernadores regionales. Una y otra norma se reproducen en términos casi idénticos en los artículos 109 y 172, respectivamente, de la Ley N 18.700 Orgánica Constitucional sobre Votaciones Populares y Escrutinios. En esa misma ley puede encontrarse además la siguiente definición de voto en blanco: "Se escrutarán como votos en blanco las cédulas que aparecieren sin la señal que indique una preferencia por candidato u opción del elector, contengan o no en forma adicional leyendas, otras marcas o señas gráficas”.

Como se puede apreciar, de las disposiciones transcritas se desprende que el ordenamiento jurídico chileno adhiere a la concepción tradicional del voto en blanco, en dos sentidos. En un primer sentido, en cuanto se le considera como un voto no expresado, por no manifestar explícitamente una preferencia electoral. En un segundo sentido, en cuanto se le priva de todo valor jurídico y se le equipara en sus efectos al voto nulo, considerándolo como no emitido.

\section{Proyecto de reforma constitucional para reconocer validez al voto en blanco}

El 3 noviembre del 2017 un grupo de parlamentarios presentó una moción que "Modifica la Carta Fundamental para reconocer validez al voto en blanco en elecciones populares" (Boletín No 11453-07) ${ }^{58}$. El proyecto incorporaría una definición de voto en blanco al artículo 15 de la Constitución, en el que se considerarían como tales "aquellos sin señal que indique una preferencia por candidato u opción" 59 . Además agregaría en el artículo 26 que "[s]i en la elección de Presidente de la República el voto en blanco obtuviere más sufragios que cualquiera de los candidatos, deberá repetirse por una sola vez la votación y no podrán presentarse los mismos candidatos”. En términos análogos, se modificaría el artículo 111 en términos tales que "[s]i en la elección de gobernador regional el voto en blanco obtuviere más sufragios que cualquiera de los candidatos, deberá repetirse por una sola vez la votación y no podrán presentarse los mismos candidatos”.

${ }^{58}$ Este es el segundo proyecto de reforma constitucional dirigido a reconocer validez al voto en blanco en Chile. El primer proyecto fue presentado el 12 de febrero de 2015, bajo el título "Proyecto de reforma constitucional referido al efecto de los votos blancos (Boletín $\mathrm{N}^{\circ}$ 7.397-07)". El proyecto, que actualmente se encuentra archivado, no concitó mayor interés académico o mediático.

${ }^{59}$ El sentido de elevar a rango constitucional la definición de voto en blanco es permitir su ulterior desarrollo y especificación a nivel legal, tanto en la Ley $N^{\circ} 18.700$ Orgánica Constitucional sobre Votaciones Populares y Escrutinios, como en la Ley No 18.695 Orgánica Constitucional de Municipalidades. 
Del texto del proyecto se desprende que este persigue tres objetivos claves. Primero, diversificar los mecanismos que permitan la expresión institucional de un mayor número de ideas (considerando 1$)^{60}$. Segundo, "permiti[r] la incidencia real de la diversidad de opiniones en torno a la vida en común” (considerando 1). Y, tercero, "permitir la expresión de disconformidad con las alternativas y candidatos sometidos a votación popular" (considerando 6). Con fundamento en estos dos considerandos se puede afirmar que los autores de la referida moción hacen suyas las premisas que a nivel académico justificarían el reconocimiento del voto en blanco, particularmente en cuanto a canalizar el descontento electoral y enviar una clara señal del mismo al sistema político.

\section{Análisis crítico del proyecto de reforma constitucional}

Como se señaló más arriba, el proyecto persigue que se reconozca a nivel constitucional la validez del voto en blanco y que se le atribuyan efectos electorales directos en las elecciones de Presidente de la República y Gobernador Regional. Sin embargo, "queda delegado en la ley correspondiente determinar los mecanismos específicos de expresión de dicho voto" (considerando $6^{\circ}$ ). Es así como un análisis crítico de la reforma propuesta requiere hacer una proyección respecto de cuáles podrían ser tales mecanismos específicos y de cómo ellos se conjugarían con otros factores institucionales y políticos. El análisis se centrará en el modo en que la reforma podría incidir en la garantía de pluralismo político, en las tasas de participación electoral y en las expectativas de autorreforma de los partidos políticos, particularmente en las elecciones presidenciales.

\section{a) Pluralismo político}

La garantía de pluralismo político (artículo $19 \mathrm{~N}^{\circ} 15$ inciso $6^{\circ} \mathrm{CPR}$ ) comprende al menos dos aspectos. En su primer aspecto, ella "exige que los más diversos puntos de vista puedan ser expresados y sostenidos en el discurso público"61. Por consiguiente, "comprende la libertad para elaborar ideas, el derecho a difundirlas y a organizarse para llevarlas a la práctica" 62 . Por otro lado, en su segundo aspecto, esta garantía también “considera que todos los partidos, grupos y tendencias políticas pueden buscar hacerse del gobierno" 63 . En este sentido, el Estado debe asegurar a esos partidos, grupos y tendencias políticas la competencia en igualdad de condiciones por el poder, absteniéndose de adoptar medidas que favorezcan injustamente a uno de ellos en desmedro de los otros. Aunque en el proyecto se enfatiza rigurosamente el modo en que el reconocer validez

60 " $[\mathrm{L}] \mathrm{a}$ democracia se protege cuando un mayor número de ideas pueden expresarse institucionalmente. Por ello, el sistema político debe velar por diversificar los mecanismos que permitan dichas formas de expresión y canalizarlas, encontrando las herramientas específicas que permitan la incidencia real de la diversidad de opiniones en torno a la vida en común”.

61 Marshall, 2011, p. 19.

62 Tribunal Constitucional, Sentencia Rol N $567-2006$, c. $22^{\circ}$.

${ }^{63}$ Marshall, 2011, p. 19; Marshall, 2008, p. 56. 
al voto en blanco incidiría en el primer aspecto del pluralismo político, no resulta del todo claro qué impacto podría tener en el segundo.

a) Una variable a tomar en cuenta al discutir las posibles repercusiones del proyecto referentes a la garantía de pluralismo político es el diseño de las papeletas de votación, en términos de la forma en que el votante expresará su voluntad de emitir un voto en blanco. La propuesta de definición de voto en blanco contenida en el proyecto ("aquel[] sin señal que indique una preferencia por un candidato o una opción") sugiere que la reforma no alteraría el diseño de las papeletas, de manera que no se incluiría explícitamente una opción de voto en blanco. De ser así, se corre el riesgo de interpretar equívocamente las motivaciones de los electores al emitir este voto, considerando la ya referida ambigüedad que encierra el solo hecho de no marcar el voto.

En cuanto a la alternativa de incluir una opción explícita, como se ha dicho, ella tendría la ventaja teórica de eliminar la ambigüedad e imprecisión que tradicionalmente se atribuye al mensaje subyacente a los votos en blanco. Gracias a ella podría descartarse, al menos en línea de principio, la posibilidad de que la preferencia expresada por el elector se deba nada más que a su apatía, confusión o ignorancia ${ }^{64}$. Efectivamente, marcar el espacio destinado específicamente al voto en blanco en la papeleta de votación - o presionar el respectivo botón en la máquina de votación, según el caso- parece ser un acto con significación más clara e inequívoca que tan solo dejar de marcarla ${ }^{65}$. Por consiguiente, se robustecería aún más la función expresiva o comunicativa del voto en blanco como instrumento para transmitir a los partidos políticos mayoritarios un indiscutible mensaje de descontento frente a sus propuestas, candidatos o integrantes.

Con todo, desde una perspectiva crítica, se ha sostenido que la práctica de incorporar una opción explícita de voto en blanco en la papeleta de votación puede tener un impacto negativo respecto de la garantía de pluralismo político. Por ejemplo, en los países post-Unión Soviética, la lógica subyacente a esta práctica era evitar que el disenso político se canalizara mediante partidos minoritarios ${ }^{66}$. Esto explicaría por qué las estadísticas electorales de países como Ucrania indican que, tras la supresión de la opción del voto "contra todos", aumentó la votación por aquellos partidos ${ }^{67}$. Por el contrario, en el estado de Nevada se ha observado que la presencia de una opción de voto en blanco no ha disminuido el apoyo por partidos minoritarios ${ }^{68}$. El que la evidencia en este punto sea inconsistente lleva a cuestionar la conveniencia de adoptar una reforma de esta envergadura, sin contar previamente con estudios acerca de las motivaciones de los votantes chilenos para emitir un voto en blanco y del modo en que contar o no con una opción explícita en la papeleta para emitirlo repercutiría en su comportamiento electoral.

b) Una variable adicional a considerar al apreciar el potencial impacto del proyecto de reforma en la garantía de pluralismo político es la existencia de restricciones respecto

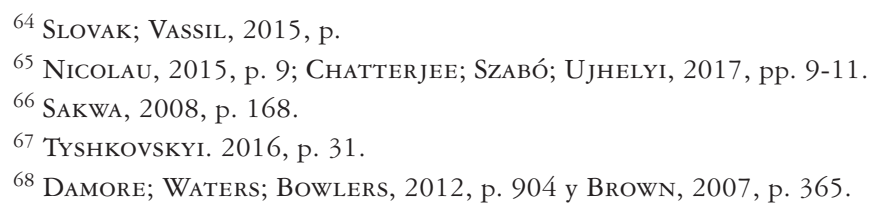


de los partidos que pueden participar en la repetición de las elecciones. Un primer supuesto sería que en la repetición de las elecciones solo pudieran participar aquellos partidos que superen un determinado umbral en la primera elección. La consecuencia práctica de estos umbrales es elevar el número total o absoluto de votos, a partir de ello se calcularía el porcentaje de votos requeridos para participar en la segunda elección. Dicho de otro modo, al incluir los votos en blanco entre los votos con los que se calcularían los umbrales, aumentaría el total de votos a computar y, naturalmente, al aumentar el total cada partido requeriría más votos a favor para poder superar el umbral. Como resultado, disminuirían las posibilidades de participación de los partidos minoritarios y se promovería la formación de pactos electorales o coaliciones, al tiempo que aumentarían las posibilidades de participación de los partidos mayoritarios ${ }^{69}$.

Un segundo supuesto, por otro lado, sería que en la repetición de las elecciones pudieran participar todos los partidos que participaron en la primera elección, sin restricciones. En este caso, aunque no opere el umbral, los partidos minoritarios podrían quedar igualmente en una situación de desventaja para presentar una nueva candidatura respecto de los partidos mayoritarios. Tan solo considerando que los aportes públicos a partidos políticos son asignados en función del número de votos válidamente emitidos que estos obtengan ${ }^{70}$ y el exiguo tiempo que -presumiblemente- transcurriría entre la primera y la segunda elección, es razonable suponer que levantar una nueva candidatura presidencial podría resultar considerablemente más difícil para esta clase de agrupaciones. Por otro lado, surge la duda respecto del tratamiento que en una eventual reforma se daría a las candidaturas independientes. Si ellas también fueran excluidas de la segunda elección, se vulneraría la garantía de igualdad entre independientes y miembros de partidos políticos, tanto en la presentación de candidaturas como en su participación en los procesos electorales (artículo 18 inciso $1^{\circ} \mathrm{CPR}$ ).

b) Participación electoral y expectativas de autorreforma de los partidos políticos

Una segunda línea de discusión se relaciona con el potencial impacto que la reforma tendría, de prosperar el proyecto, en las tasas de participación electoral. Si bien el aumento de estas no es uno de los objetivos declarados del proyecto bajo análisis, en un país con tasas de abstención superiores al 50\%, este parece ser un aspecto digno de consideración.

De momento, no existe evidencia empírica sólida en torno a la existencia de una correlación positiva entre el reconocimiento del voto en blanco y aumento de las tasas de participación. Si bien existen investigaciones que avalan esta idea (una, en particular, sobre el caso de la India $)^{71}$, otras parecen negarla. Así, por ejemplo, se ha observado que las tasas de participación electoral en el estado de Nevada han disminuido considerablemente con

69 Baskaran; Lopes da Fonseca, 2016, p. 135; Capoccia, 2002, pp. 180-183; Mora, 2012 , pp. 72 y 77 .

${ }^{70}$ Artículo 33 bis inciso $5^{\circ}$ de la Ley $N^{\circ}$ 18.603, Ley Orgánica Constitucional de los Partidos Políticos.

${ }^{71}$ Ibid., pp. 21-24 y 42-50. 
el tiempo, pese a que la opción "ninguno de estos candidatos" se encuentra disponible en las papeletas de votación desde $1975^{72}$. Al contrario, se observa que "después una ráfaga de interés por NDEC, los votantes dejaron de usar la opción"73. En Ucrania, por su parte, donde el voto "contra todos" estuvo vigente desde 1994 hasta su abolición en el 2012, tampoco se apreció una disminución de las tasas de participación respecto de procesos electorales previos. En lugar de ello se produjo un aumento de la votación por partidos minoritarios ${ }^{74}$. Por último, en una investigación respecto de los resultados de las elecciones generales en España durante el periodo comprendido entre 1982 y 1986 se concluyó que el aumento del voto en blanco era muy superior al crecimiento del censo. A causa de que no había correlación entre la tasa de abstención y el incremento de los votos en blanco, era plausible conjeturar que quienes eligieron la opción en comento no eran necesariamente nuevos votantes que antes se abstenían, sino votantes antiguos que simplemente cambiaron su voto ${ }^{75}$.

$\mathrm{Ni}$ siquiera en Colombia, donde el voto en blanco tiene influencia decisiva en los resultados de la elección, existe evidencia significativa en relación con este punto. Aunque allí el voto en blanco ha aumentado (e incluso obtenido algunas notables victorias electorales ${ }^{76}$ ) desde que se le reconociera validez en 1991, en las últimas elecciones la abstención también ha experimentado un crecimiento considerable ${ }^{77}$. Lo anterior significa que ni siquiera en aquellos sistemas en que el voto en blanco produce efectos electorales directos puede darse por establecida sin más una relación directa entre el reconocimiento jurídico de este y la disminución de las tasas de abstención.

Más allá de estos antecedentes, ahora desde un punto de vista teórico, existen buenas razones para cuestionar la idea de que el reconocimiento jurídico del voto en blanco por sí solo pueda conllevar una disminución de las tasas de abstención electoral. Más aún, existen buenas razones para suponer que, lejos de constituir la solución a la crisis de alienación política que aqueja a buena parte de las democracias contemporáneas, la institucionalización del voto en blanco podría terminar por agudizarla. Dichas razones se vinculan con la discrepancia que puede existir entre las expectativas de quienes emiten un voto en blanco en orden a influir en el sistema político y los genuinos efectos que este produce o puede producir.

Efectivamente, el reconocimiento del voto en blanco como voto escrutable pero no computable a efectos de adjudicar representación suele encerrar una paradoja que conviene tener presente. Entendido el voto en blanco como voto protesta, podría existir una contradicción entre los fines conscientes que perseguiría el votante con su emisión y los fines que efectivamente podría producir, adulterándose así la genuina voluntad de

\footnotetext{
72 Brown, 2011, p. 365.

${ }^{73}$ En palabras del Secretario de estado de California: "after a brief flurry of interest in NOTA, voters stop using the option and candidates continue behaving as before". Citado en DAMORE; WATERs; Bowlers, 2012, p. 897.

74 Tyshkovskyi, 2016, p. 30.

75 Bobillo, 1988, pp. 79-81.

${ }^{76}$ LozAnO; Rodríguez, 2015, p. 138.

${ }^{77}$ Ibid.
} 
aquel. Acerca de este asunto, aunque específicamente a propósito del sistema electoral español, se ha dicho que: "aquellos electores que voten en blanco, en realidad, lo que están haciendo - de manera no voluntaria- no es otra cosa que diezmar las posibilidades de terceras formaciones a las que ni siquiera han tenido en cuenta a la hora de formar su decisión, beneficiando de forma muy apreciable a aquellas a las que en verdad sí han decidido castigar" 78 .

A mi juicio, esta observación también podría ser válida para la propuesta de reforma al sistema electoral chileno. Y es que el voto en blanco solo es apto para manifestar disenso o no adhesión a los candidatos o partidos en competencia, pero carece de contenido propositivo en sí mismo. Este jamás puede adjudicar representación y, por ende, resultar "vencedor". Aun cuando se repitan las elecciones, tarde o temprano tendrá que conducirse un segundo proceso electoral que sí concluya en la designación de representantes, en el que el voto en blanco ya no podrá tener efectos electorales directos. Por lo demás, un inicial "triunfo" del voto en blanco no evita que los mismos partidos o pactos electorales contra los que se manifestó insatisfacción sigan participando en la repetición de esta. Naturalmente, tampoco evita que esos mismos partidos sigan participando en otros procesos electorales futuros.

Para este segundo proceso deberán inscribirse nuevas candidaturas, en un plazo relativamente breve (como referencia, en Colombia el plazo es de diez días corridos contados a partir del día siguiente a la declaratoria de resultados por la correspondiente autoridad escrutadora). De ahí que surjan serias dudas respecto de la factibilidad de permitir la participación de la ciudadanía en la designación de los nuevos candidatos, mediante elecciones primarias. Siendo así, cabría conjeturar que los candidatos serán elegidos entre (i) los precandidatos derrotados en los procesos de designación de candidatos oficiales de cada partido o coalición, u (ii) otros personajes ya prominentes de dicho partido o coalición. Cabría igualmente suponer, considerando el potencial impacto negativo de la atribución de efectos electorales directos al voto en blanco en la participación de los partidos minoritarios y de candidatos independientes, que los partidos mayoritarios tendrían una probabilidad más alta de ser los vencedores de todas formas ${ }^{79}$.

De ser correctas estas suposiciones, sería poco realista esperar que una eventual implementación del proyecto conduzca a la autorreforma de los partidos políticos o al incremento de su responsiveness frente a las expectativas de la ciudadanía. Antes bien, las reflexiones aquí plasmadas sugieren que el reconocimiento jurídico del voto en blanco, en los términos propuestos en el proyecto, podría favorecer a los partidos mayoritarios y conducir a la preservación del statu quo, en perjuicio de los partidos minoritarios y de las candidaturas independientes.

Esta observación no debería causar sorpresa alguna. Al fin y al cabo, como se indicó más arriba, la emisión de un voto en blanco no implica rechazo hacia el sistema

\footnotetext{
78 MORA, 2012, p. 77.

${ }^{79}$ BRICHARD, 2014, p. 2.
} 
democrático en sí mismo. Por el contrario, implica al mismo tiempo rechazo hacia las opciones electorales disponibles en un momento determinado y una adhesión a las formas ortodoxas de participación en democracia. De esta suerte, en cuanto instrumento de protesta política, la emisión de un voto en blanco resulta particularmente débil a efectos de producir cambios estructurales en el sistema de partidos ${ }^{80}$. Por esta razón, aun reconociendo como valiosa la posibilidad de que los votantes dejen en blanco su papeleta con fines puramente expresivos, la propuesta de institucionalizar el voto en blanco y asignarle efectos electorales directos debe ser manejada con cautela.

\section{CONCLUSIÓN}

1. En esta investigación se ha abordado la creciente tendencia a ver el voto en blanco como un tipo de voto de protesta y una expresión de disenso político. Dicha tendencia se puede observar no solo a nivel académico o teórico, sino que se refleja también en la práctica de diversos sistemas electorales en orden a dar algún tipo de reconocimiento al voto en blanco. El interés por este tema encuentra tres justificaciones. La primera de ellas dice relación con las virtudes o efectos positivos que se atribuyen a la antes referida práctica, en términos de su potencial para promover la libertad de expresión, disminuir las tasas de abstención electoral y fortalecer la gestión de los partidos políticos. La segunda de ellas tiene que ver con colmar un vacío en la literatura nacional respecto de este asunto. Y la tercera de ellas apunta a proporcionar un análisis crítico del proyecto de reforma constitucional recientemente presentado por un grupo de parlamentarios, que aspira a la institucionalización del voto en blanco en Chile.

2. Bajo el rótulo de reconocimiento del voto en blanco se agrupan varias modalidades de institucionalización o valoración jurídica del voto en blanco, existentes a nivel comparado. Las mismas han sido sistematizadas en tres categorías. Primero, la incorporación formal de una opción de voto en blanco en las papeletas de votación y la presentación de candidaturas o partidos que simulen esa incorporación. Segundo, la consideración del voto en blanco en el cálculo de umbrales para efectos de permitir la participación de partidos políticos en procesos electorales, asignar tiempos de propaganda política en medios de comunicación de titularidad pública o de distribuir recursos públicos para el financiamiento de campañas políticas. Y, tercero, la atribución de efectos jurídicos directos al voto en blanco en la adjudicación de representación.

3. Una de las principales conclusiones de esta investigación es que el proyecto de reforma constitucional para el reconocimiento de la validez del voto en blanco puede producir efectos objetables a la luz de la garantía de pluralismo político y de su incidencia en la participación electoral. Más concretamente, se pone de relieve el modo en que la reforma propuesta podría contribuir a preservar el statu quo y favorecer a los partidos mayoritarios, así como a desfavorecer o excluir a partidos minoritarios y candidatos 
independientes. Es así como, eventualmente, al reconocer validez al voto en blanco se podrían producir efectos contrarios a los que, según los propios promotores del proyecto, buscarían los votantes que deciden emitirlo en señal de protesta política.

4. Para terminar, se plantea un cuestionamiento al potencial de la propuesta contenida en el proyecto para canalizar adecuadamente la protesta política de los electores y para contribuir a la autorreforma del sistema de partidos. El cuestionamiento se funda en dos ideas centrales. Primero, en el eventual impacto negativo de la reforma respecto de la garantía de pluralismo político y en la participación electoral. Segundo, en el contrasentido de manifestar eficazmente insatisfacción con las opciones que ofrece el proceso electoral por medio de uno de los más convencionales instrumentos dirigidos a manifestar apoyo al mismo, en una democracia representativa. De resultas, aunque el cómputo de los votos en blanco emitidos en cada elección pueda ser útil como indicador de insatisfacción electoral, es altamente dudoso que la atribución de validez al voto en blanco pueda traducirse en una reforma significativa del sistema.

\section{BIBLIOGRAFÍA}

Aldashev, Gani; Mastrobuoni, Giovanni, "Invalid Ballots and Electoral Competition”, Political Science Research and Methods (2016), pp. 1-22.

Álvarez, R. Michael, D. Kiewiet, Roderick; NúÑez, Lucas, “A Taxonomy of Protest Voting”, Annual Review of Political Science 21 (2018), pp. 1-28.

Baskaran, Thushyanthan; Lopes Da Fonseca, Mariana, "Electoral thresholds and political representation", Public Choice 169 (2016), 117-136

Biblioteca del Congreso Nacional de Chile, Historia de la Ley. Constitución Política de la República de Chile de 1980. Artículo 15. Derecho de Sufragio. Recuperado el 01.11.2017 de: www.leychile.cl/Navegar/scripts/obtienearchivo?id = recursoslegales/10221.3/367/4/ HLArt15CPR.pdf.

Boвbio, Norberto, Teoría general de la política, Trotta, Madrid, 2003.

BobILlo, Francisco J., "El voto estéril en las elecciones generales españolas", Revista de Estudios Políticos (Nueva Época) 62 (1988), pp. 69-88.

Brennan, Geoffrey; Hamlin, Alan, "Expressive voting and electoral equilibrium", Public Choice 95 (1998), pp. 149-175.

Brewer-Carías, Allan, "La nulidad de los actos electorales: Una perspectiva constitucional comparada", en IIDH/CAPEL, Transición democrática en América Latina: reflexiones sobre el debate actual. Memoria III Curso Anual Interamericano de Elecciones, IIDH/CAPEL, San José, 1990, pp. 89-137.

Brichard, Nathalie, Blank Vote in the Upcoming Elections, Mission of Electoral Observation MOE Colombia, 2014. Recuperado el 01.11.2017 de: http://www.gndem.org/sites/default/files/ Article_5_Blank_Votes\%20(1).pdf.

Brown, Adam R., "Losing to nobody? Nevada's 'none of these candidates' ballot reform”, The Social Science Journal 48 (2011), 2, pp. 364-370.

Capoccia, Giovanni, "The Political Consequences of Electoral Laws: The German System at Fifty", West European Politics 25 (2002), 3, pp. 171-202.

Cea Egaña, José Luis, Derecho constitucional chileno, Tomo III, Santiago, Ediciones Universidad Católica de Chile, 2013. 
Chatterjee, Somdeep; Szabó, Andrea; Ujhelyi, Gergely (25 de octubre de 2017), "None Of The Above Votes in India and the Consumption Utility of Voting". Recuperado el 30.12.2017, de: http://www.uh.edu/ aszabo2/nota12.pdf.

Damore, David F.; Waters, Mallory M.; Bowler, Shaun, "Unhappy, Uninformed, or Uninterested? Understanding 'None of the Above' Voting”, Political Research Quarterly 65 (2012) 4, pp. 895-907.

Downs, Anthony, An Economic Theory of Democracy, Harper \& Row, New York, 1957.

Driscoll, Amanda; Nelson, Michael J., "Ignorance or Opposition? Blank and Spoiled Votes in Low-Information, Highly Politicized Environments”, Political Research Quarterly (2014), pp. 1-15.

Engelen, Bart, "Why Compulsory Voting Can Enhance Democracy", Acta Politica 42 (2007), pp. $23-39$.

Galatas, Steven, “'None of the Above' Casting Blank Ballots in Ontario Provincial Elections”, Politics E Policy 36 (2008), 3, pp. 448-473.

Hernández Bravo, Juan, “Los sistemas electorales”, en Del Águila, Ramón (dir.), Manual de Ciencia Política, Trotta, Madrid, 2008, pp. 349-390.

Hillman, Arye L., "Expressive behavior in economics and politics", European Journal of Political Economy 26 (2010), 4, pp. 403-418.

Hooghe, Marc; Marien, Sofie; Pauwels, Teun, "Where do Distrusting Voters Turn to if there is no Viable Exit or Voice Option? The Impact of Political Trust on Electoral Behaviour in the Belgian Regional Elections of June, 2009”, Government and Opposition. International Journal of Comparative Politics 46 (2011), 2, pp. 245-273.

Ibarzabal, Nora; Laruelle, Annick, "Ghost Seats in the Basque Parliament", European Journal of Operational Research (2017), pp. 1-10.

Lozano García, Mario Alexander; Rodríguez Casallas, Diego F., "Voto en blanco, voto nulo y abstención: expresión de rechazo de la ciudadanía boyancense, en los comicios parlamentarios”, Revista Vía Iuris 18 (2015), pp. 135-150.

Marshall Barberán, Pablo, "Notas sobre el contenido del principio de la democracia", Revista Chilena de Derecho y Ciencia Politica 2 (2011), 1, pp. 9-36.

Marshall Barberán, Pablo, "Partidos e independientes ante la constitución: el caso de la distribución del tiempo en la franja electoral", Revista de Derecho (Valdivia) 21 (2008), 1, pp. 45-60.

McMurray, Joseph, "Voting as communicating: Mandates, multiple candidates, and the signaling voter's curse”, Games and Economic Behavior 102 (2017), pp. 199-223.

Mora Molina, Juan Jesús, "El sistema electoral español, una propuesta realista", Anales de la Cátedra Francisco Suárez 46 (2012), pp. 69-92.

Nicolau, Jairo, "Impact of Electronic Voting Machines on Blank Votes and Null Votes in Brazilian Elections of 1998”, Brazilian Political Science Review 9 (2015), 3, pp. 3-20.

NúÑEZ, Lucas, "Expressive and Strategic Behavior in Legislative Elections in Argentina", Political Behavior 38 (2016), pp. 899-920.

SAKwA, Richard, Russian Politics and Society, Routledge, New York, 2008.

Schepis, Giovanni, "Analisi statistica dei risultati”, en Spreafico, Alberto, La Palombara, Joseph (a cura di), Elezioni e comportamento politico in Italia, Edizioni di comunitá, Milán, 1963, pp. 329-406.

SlovaK, Mihkel; VassiL, Kristjan, "Indifference or Indignation? Explaining Purposive Vote Spoiling in Elections”, Journal of Elections, Public Opinion and Parties 25 (2015), 4, pp. 463-481.

SuperTi, Chiara (13 de marzo de 2015), "Vanguard of Discontent: Comparing Individual Blank Voting, Mobilized Protest Voting, and Voting Abstention”. Recuperado el 30.12.2017, de 
Department of Government, Harvard University: http://scholar.harvard.edu/files/csuperti/ files/dissertationpaper1_spainitaly_0.pdf.

Troumpounis, Orestis, Electoral systems and forms of abstention, Tesis presentada ante el Departament d'Economia i d'Història Econòmica de la Universitat Autònoma de Barcelona, en cumplimiento parcial de los requisitos para optar al grado de Doctor en Economía. Disponible en: http://digital.csic.es/bitstream/10261/46323/1/tesis-Orestis-Troumpounis.pdf.

Tyshrovskyi, Anton, Does NOTA Option Availability Affect Voting in a Polarized Environment? Evidence from Ukranian Parliamentary Elections 2006-2012, Tesis presentada ante la Central European University, en cumplimiento parcial de los requisitos para optar al grado de Master of Arts in Economic Policy on Global Markets. Disponible en: www.etd.ceu.hu/2016/ tyshkovskyi_anton.pdf.

UGGLA, Fredrik, "Incompetence, Alienation, or Calculation? Explaining Levels of Invalid Ballots and Extra-Parliamentary Votes", Comparative Political Studies 41 (2008), 8, pp. 1141-1164.

Urdánoz Ganuza, Jorge, "Un nuevo sistema electoral”, Revista de Estudios Políticos (Nueva Época) 126 (2004), pp. 288-319.

Zulfikarpasic, Adélaïde, "Le vote blanc: abstention civique ou expression politique", Revue française de science politique 1 (2001), 51, pp. 247-268.

Jurisprudencia citada

Corte Constitucional (Colombia), sentencia de fecha 23 de junio de 2011, C-490-2011.

Corte Suprema de la India, People's Union for Civil Liberties v. Union of India (2013) (10) S.C.C., sentencia de fecha 27 de septiembre de 2013.

Tribunal Constitucional (Chile), sentencia de fecha 02 de junio de 2010, Rol No 567-2006.

Normas jurídicas citadas

Código Electoral. Publicado en el Diario Oficial, tomo 318, El Salvador, con fecha 25 de enero de 1993.

Código Electoral Colombiano (Decreto 2241 de 1986). Publicado en el Diario Oficial No 37571 , Colombia, con fecha 01 de agosto de 1986.

Código Electoral Nacional (Ley N $\mathrm{N}^{\circ}$ 19.945). Publicado en el Boletín Oficial, Argentina, con fecha 19 de diciembre de 1972.

Constitución Política de Colombia de 1991. Publicada en la Gaceta Constitucional, Colombia, $\mathrm{N}^{\circ} 114$, con fecha 04 de julio de 1991.

Constitución Política de la República de Chile. Publicada en el Diario Oficial, Chile, con fecha 22 de septiembre de 2005.

Constitución Política del Perú de 1993. Publicada en el Diario Oficial el Peruano, Perú, con fecha 30 de diciembre de 1993.

LEY 130 de 1994 (Estatuto Básico de los partidos y movimientos políticos). Publicada en el Diario Oficial No 41280, Colombia, con fecha 23 de marzo de 1994.

Ley Electoral. Publicada en La Gaceta No 168, Nicaragua, con fecha 04 de septiembre de 2012.

Ley General de Instituciones y Procedimientos Electorales. Publicada en el Diario Oficial de la Federación, México, con fecha 23 de mayo de 2014.

Ley $\mathrm{N}^{\circ}$ 7.812, Ley de Elecciones. Publicada en el Diario Oficial, tomo LXXVIII, $\mathrm{N}^{\circ} 5691$, Uruguay, con fecha 16 de enero de 1925.

Ley N 18.695 Orgánica Constitucional de Municipalidades. Publicada en el Diario Oficial, Chile, con fecha 06 de mayo de 1988. 
Ley N 18.700 Orgánica Constitucional sobre Votaciones Populares y Escrutinios. Publicada en el Diario Oficial, Chile, con fecha 26 de julio de 2006.

LEy Orgánica 5/1985, de 19 de junio, del Régimen Electoral General. Publicada en el Boletín Oficial del Estado $\mathrm{N}^{\circ} 147$, España, con fecha 20 de junio de 1985.

Ley N ${ }^{\circ}$ 18.603, Ley Orgánica Constitucional de los Partidos Políticos. Publicada en el Diario Oficial, Chile, con fecha 23 de marzo de 1987.

Ley Orgánica de Elecciones (Ley No 26859). Publicada en el Diario Oficial el Peruano, Perú, con fecha 01 de noviembre de 1997.

Ley Orgánica de Procesos Electorales. Publicada en la Gaceta Oficial No 5928E, Venezuela, con fecha 12 de agosto de 2009.

Nevada Revised Statutes (2015). Publicada en https://www.leg.state.nv.us/NRS/ (versión oficial electrónica), Estados Unidos.

Modifica la Carta Fundamental para reconocer validez al voto en blanco en elecciones populares, Boletín $\mathrm{N}^{\circ} 11453-07$.

Proyecto de reforma constitucional referido al efecto de los votos blanco, Boletín $\mathrm{N}^{\circ}$ 7.397-07. 